\title{
IoT BASED SMART AND EFFICIENT WIND TURBINE MONITORING SYSTEM
}

\author{
S. KALAIVANI \\ Research Scholar \\ Department of Electronics \& \\ Communication Engineering \\ B.S. Abdur Rahman Crescent Institute \\ of Science \& Technology \\ Chennai, India \\ skalaivani@crescent.education
}

\author{
C. THARINI \\ Professor \& Head of the \\ Department of Electronics \& \\ Communication Engineering \\ B.S. Abdur Rahman Crescent \\ Institute of Science \& Technology \\ Chennai, India \\ ctharini@crescent.education
}

\author{
A.M. AZARUDEEN, \\ R. KARTHIKEYAN \\ B. Tech. students \\ Department of Electronics \& \\ Communication Engineering \\ B.S. Abdur Rahman Crescent \\ Institute of Science \& Technology \\ Chennai, India \\ azarchn.deen@gmail.com; \\ rkarthik96.chn@gmail.com
}

\begin{abstract}
Wind Turbine industry has the improved latest generation of Wind turbines with bigger flexible blades, high tower, Good efficiency \& low cost repairing in all platforms of wind turbines from Small wind mills to Ocean wind turbines. The Control centre is responsible for Monitoring and Controlling wind turbines in wind power farms. Various parameters like Oil level, Gas leakage, air pressure, vibrations \& linear velocity, environmental condition like rain \& humidity are to be monitored and controlled for proper working of the wind turbines. In the proposed work, smart and efficient turbine network architecture is designed to automate this process. The aim of the proposed work is to monitor the different parameters of the turbine using respective sensors. The acquired sensor data are uploaded to the cloud via WiFi module for online monitoring and further data analysis. IFTTT Server of Adafruit io cloud is used to send the warning notification of the critical sensor value to the concerned person. Also the sensor node life time is taken care by implementing a proposed compression algorithm in each node that reduces the amount of data transmitted and thereby the energy consumed during transmission.
\end{abstract}

Keywords: Wind Power Farms(WPF), Internet of Things(IoT), Cloud storage, Wireless Sensor Node(WSN), Arduino UNO, Data Compression and Node life time.

\section{INTRODUCTION}

Wind turbine equipment shown in figure 1 converts the wind's mechanical energy into electrical energy [1]. Wind Turbine includes different parts such as Rotor, Transformer, Nacelle, Yaw Position, Sensor Node-ID, various sensors etc. Sensors installed in the turbine are Humidity sensor, Temperature sensor, Accelerometer, Gyroscope, Barometer, Ultrasonic Sensor for measuring oil level in the container of disc break that controls the speed of rotor blades when high speed wind blows inside the nacelle and Gas Sensor for fault detection and condition monitoring in the nacelle $\&$ rotor systems of the turbine [2].

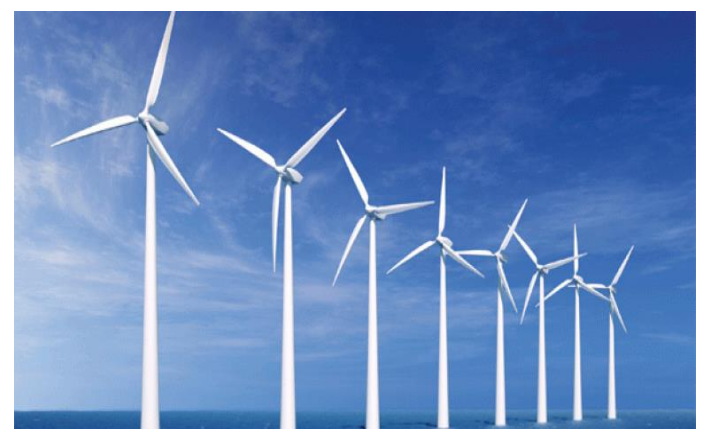

Fig. 1 Wind Turbines

Nowadays wind power industries are equipped with Wind Turbines of longer \& broad flexible blades, tall tower, better efficiency and low cost repairing 
system.

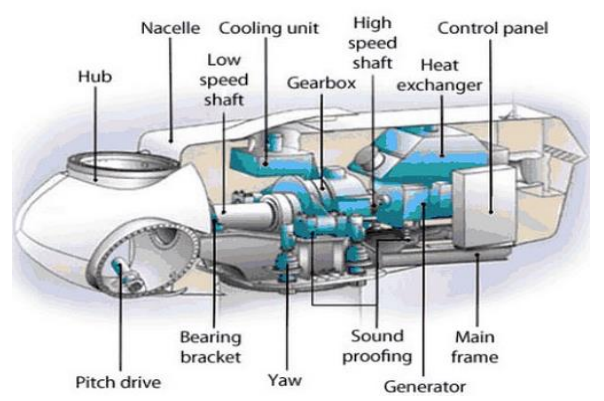

Fig. 2 Nacelle box

The control centre is responsible for the analysis of parameters obtained from the sensors installed in the Nacelle box of Wind-turbines at Wind Power farms [3]. The internal structure of the nacelle box is shown in figure 2. Suitable communication network architecture is designed for communicating and sharing the sensed data.

The current technology used in the field of wind turbines to control and monitor the parametric data is very cost effective due to the construction of network architecture with optical fibre cables. Also there is no availability of dedicated server to receive the output data from the sensors installed for ubiquitous turbine health monitoring. The proposed module is an IoT based system [4] used to overcome these problems that consist of Arduino UNO board interfaced with various sensors [5] and the ESP8266 NODE MCU $\mathrm{WiFi}$ Module. The sensor data is shared via $\mathrm{WiFi}$ module to Adafruit IO web IDE cloud storage webpage for further data analysis. IFTTT Server of Adafruit IO Cloud is mainly used to send warning notification of main critical sensors to Android mobile to avoid major accident.

\section{PROPOSED SYSTEM}

The proposed system consists of different sensors like Ultrasonic sensor, Rain sensor, Gas sensor, Temperature sensor, Barometric sensor and Accelerometer \& Gyro sensor. The other harwares and software involved in the proposed system are Arduino R3 UNO, Adafruit IO Cloud storage, NODE MCU to make communication, IFTTT Server to receive notification at faster rate than the existing system. The figure 3 shows the general block diagram of the proposed system.

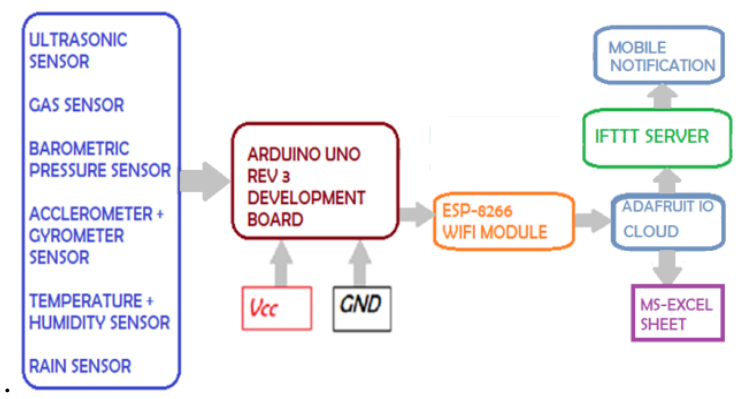

Fig. 3 Block diagram of Proposed System

The proposed system is used to detect the values of parametric sensors in the nacelle of the wind turbines and to analyze the data periodically through logging into cloud storage web server whenever required. The ubiquitous monitoring system also alerts the control center for the critical values of the sensors.

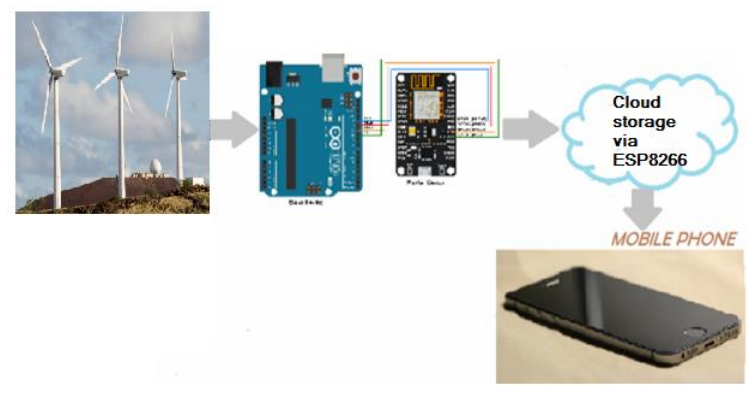

Fig. 4 Schematic Diagram of Proposed System

The above figure 4 shows the schematic diagram of the proposed system that clearly picturize the interfacing of Arduino UNO board to the cloud server via the $\mathrm{WiFi}$ module and the critical data transfer from cloud to the Android mobile App.

\section{HARDWARE DESCRIPTION}

The various hardware components used in the proposed system are described in this section.

\subsection{Arduino UNO R3}

Arduino is an open source PC hardware and software Program Company [7] that designs and manufactures single microcontrollers and microcontroller kits for building digital devices and interactive projects that can sense and control objects in the physical and digital world. The 
Arduino Uno R3 shown in the figure is a microcontroller board based on the Atmel's ATmega328.

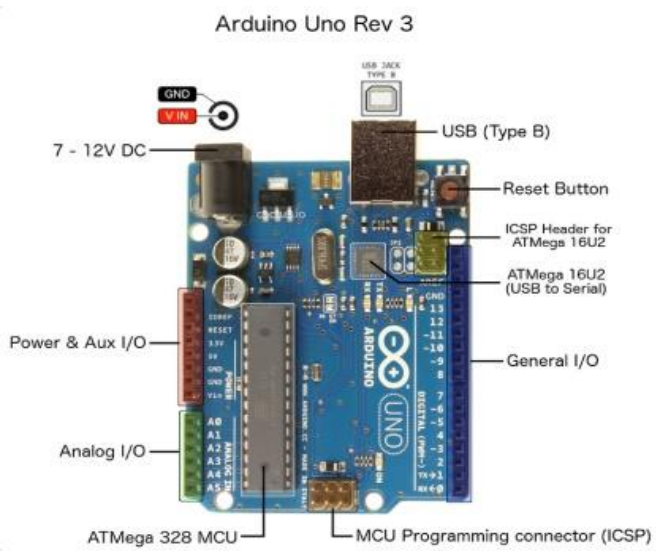

Fig.5 Arduino UNO R3 Pinout Diagram

It consists of 14 digital I/O pins and 6 analog inputs. The board is powered through USB connection or with an external power supply. It is easily connected to a computer using a USB cable

\subsection{Ultrasonic Sensor}

The Ultrasonic sensor consists of a transmitter and a receiver [10]. Its working principle is as shown in figure 6 . The transmitter transmits ultrasonic wave that gets reflected by the interfering object and is received back by the receiver in the sensor. With respect to the received signal strength the distance between the sensor and the object is calculated.

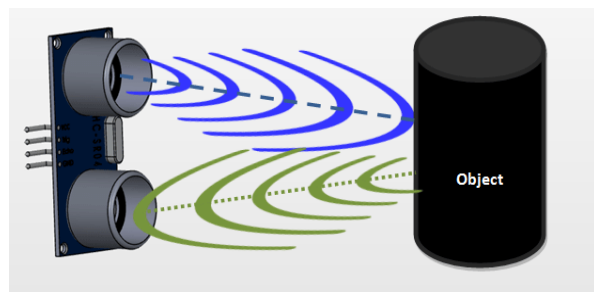

Fig. 6 Ultrasonic Working Principle

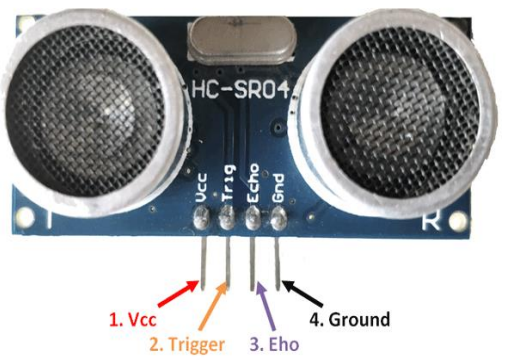

Fig. 7 4HC-SR04 Pin Diagram
The ultrasonic sensor 4HC-SR04 shown in figure 7 is used for oil level monitoring in the proposed system.

\subsection{Gas Sensor}

The gas sensors are used in applications that involve air quality monitoring, alcohol/smoke detection, gas leakage detection etc [8].
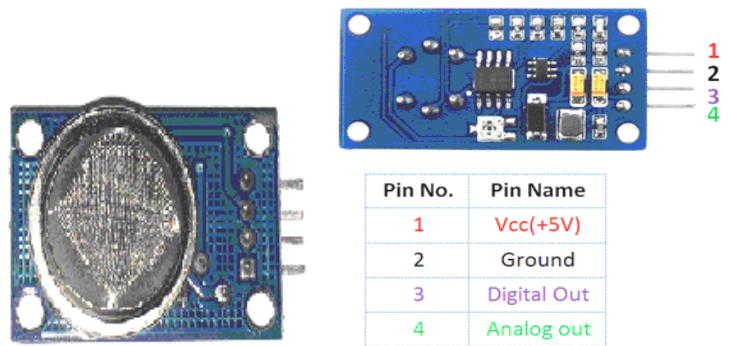

Fig. 8 MQ-135 Module \& its pin details

The MQ-135 Gas sensor shown in the figure 8 is interfaced in the proposed system and is suitable for detecting smoke \& measuring air quality.

\subsection{Gyro Accelerometer Sensor}

The accelerometer is used to measure the linear acceleration on three axes and gyroscope measures the angular velocity. MPU6050 is a Micro ElectroMechanical Systems (MEMS) [10] which consists of a 3-axis Accelerometer and 3-axis Gyroscope is shown in figure 10 .

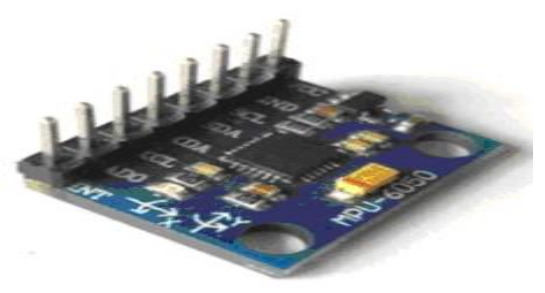

Fig. 9 MPU6050 Module

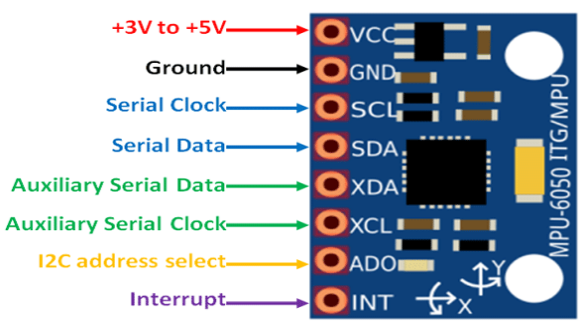


Fig. 10 MPU6050 Pin Diagram

The pin diagram of MPU6050 is shown in figure 10 . It is used to monitor vibrations in the rotating components of the output shafts and to detect the blade tip deflection.

\subsection{Humidity Sensor}

The DHT11 shown in figure 11 is a basic digital temperature and humidity sensor. The operating voltage is $3.3 \mathrm{~V}$ or $5 \mathrm{~V}$ DC [12]. It measures the temperature range of about $0-50^{\circ} \mathrm{C}$ and relative humidity of about $20-95 \%$.

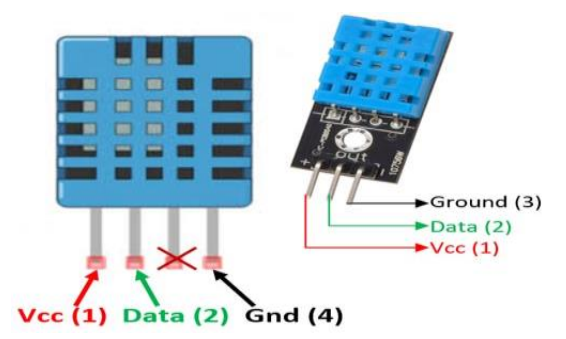

Fig.11 DHT11 sensor Pin Diagram

The sensor is easily interfaced with the Arduino board and is used to monitor the temperature \& humidity.

\subsection{Barometric Sensor}

Barometric Sensor is used to measure the atmospheric pressure. BMP180 shown in figure 12 is a low cost barometric air pressure sensor [13].

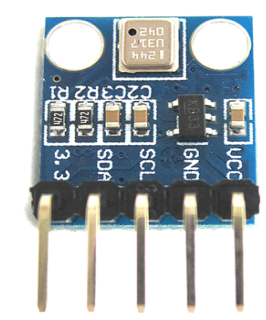

Fig. 12 BMP180 Sensor

It is used to measure the pressure of air that helps for weather monitoring.

\subsection{Rain Sensor}

The Rain sensor shown in the figure 13 is used to measure the moisture level in the atmosphere [12]. It is activated by the rainfall.

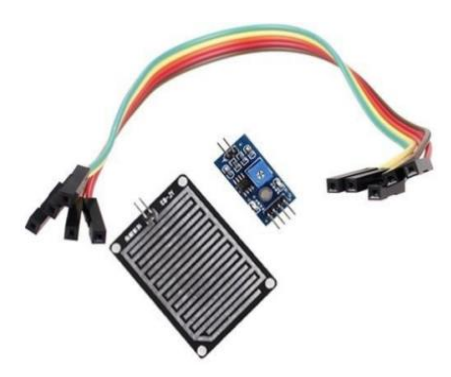

Fig. 13 Rain Sensor

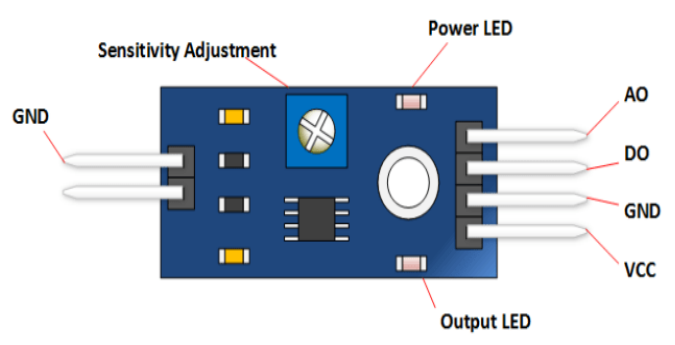

Fig. 14 MS554 Pin Diagram

The MS554 rain sensor shown in figure 14 used in the system helps in detecting the rain fall, as the turbine blades working under different environmental condition are subjected to acute vibrations and irregular distortions.

\subsection{Node MCU ESP-8266}

ESP8266 node MCU module shown in figure 15 is a firmware that includes a built-in Wi-Fi SoC with reliable overall performance among various applications in IoT [14].

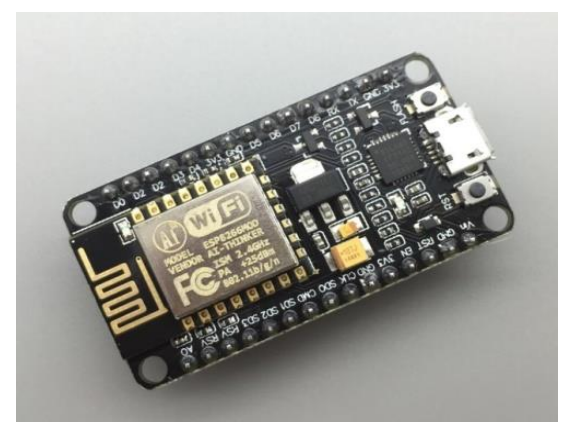

Fig. 15 ESP8266 Module

It is easily interfaced with the Arduino UNO board and thereby provides a great connection to the cloud. 


\section{SOFTWARE DESCRIPTION}

\subsection{Arduino Software IDE}

Arduino Integrated development environment (IDE) is an open source and cross-platform application with a text editor to write the code and upload it to the Arduino board [15]. Programs written on the Arduino Software (IDE) are known as sketches. These sketches are written with the textual content and are dumped into the microcontroller. The toolbar buttons allow the user to verify and upload programs, create, open, or save sketches, or start the gradual monitor. The errors in the program are displayed while compiling for further corrections. Once error is corrected the program is uploaded to the controller in the Arduino board. The output of the program is seen by accessing the serial port display.

\subsection{Adafruit Server}

The Adafruit Web IDE is easy to connect with MCU (Microcontroller Unit) boards like Raspberry Pi or BeagleBone or Arduino or any other microcontroller board with WiFi module [16]. The Web IDE helps in accessing our own web server created for online monitoring of data.

\subsection{IFTTT Notification Applet}

IFTTT, an acronym for If This Then That, is a free service that automates the connectivity between apps and services [17]. It enables the users to utilize connectivity between Internet-connected services by creating chains of simple conditional statements that are triggered based on changes to other web services.

\section{EXPERIMENTAL RESULTS}

The experimental set-up of the proposed system is shown in the below figure 16 .

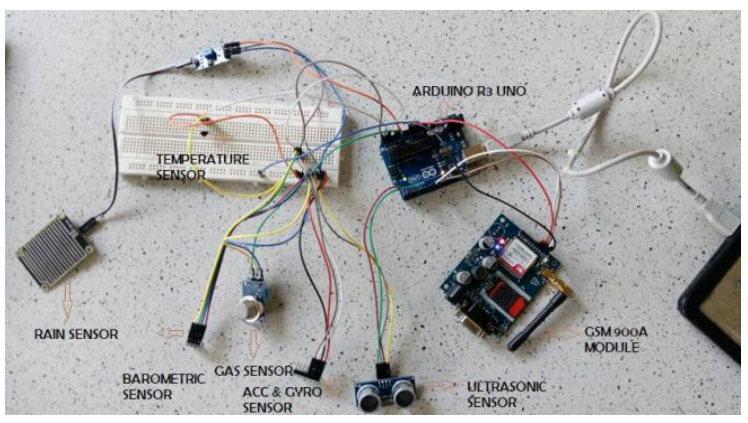

Fig. 16 Experimental setup
The different sensors discussed in the section 3 are interfaced with the Arduino UNO board which in turn is connected to the ESP8266 WiFi module.

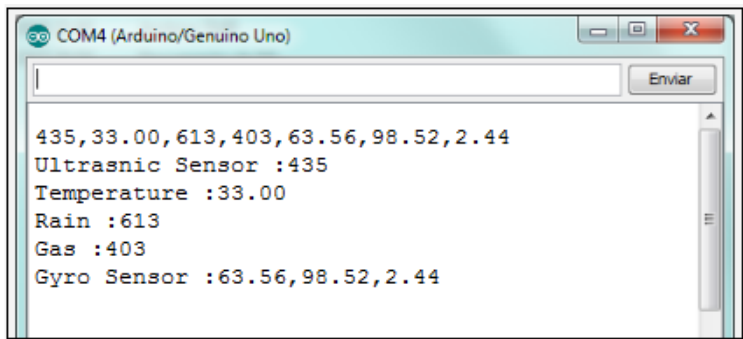

Fig. 17 Arduino Serial port Output

Once the sensor data are acquired they are displayed in the serial port of the Arduino IDE as shown in the figure 17. Each sensor are provided with an unique ID like ultrasonic sensor is referred as sensor 1, temperature sensor as sensor 2, gas sensor as sensor 3 , gyro sensor as sensor 4 , accelerometer with three axes values as sensor 5,6 \& 7, and rain sensor as sensor 8. Each sensor values are the transmitted to the webpage created via Adafruit server and the data are displayed in the excel sheet as shown in the figures $18-22$.

\begin{tabular}{|c|c|c|c|c|c|c|}
\hline 4 & A & B & C & D & $E$ & $\mathrm{~F}$ \\
\hline 3 & ODVWFYM & 27 & 800805 & 2018-05-01 & $17: 40: 58 \mid$ & UTC \\
\hline 4 & ODVWFYZ: & 24 & 800805 & 2018-05-01 & $17: 41: 08$ & UTC \\
\hline 5 & ODVWFZ2' & 204 & 800805 & 2018-05-01 & $17: 41: 18$ & UTC \\
\hline 6 & ODVWFZ5: & 21 & 800805 & 2018-05-01 & $17: 41: 28$ & UTC \\
\hline 7 & ODVWXZ4 & 5082 & 800805 & 2018-05-02 & 06:44:19 L & UTC \\
\hline 8 & ODVWXZ7. & 4936 & 800805 & 2018-05-02 & $06: 44: 28 \mathrm{~L}$ & UTC \\
\hline 9 & ODVWXZA & 43 & 800805 & 2018-05-02 & 06:44:38 & UTC \\
\hline
\end{tabular}

Fig. 18 Sensor 1 Excel sheet Data

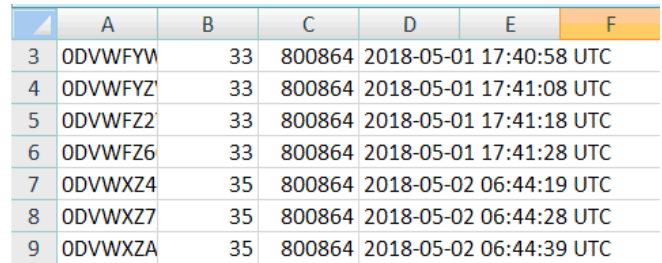

Fig. 19 Sensor 2 Excel sheet Data

The above figures show the data acquired from the ultrasonic and temperature sensors in the excel sheet of the webpage in the cloud storage. The column B of the excel sheet is the data values of the sensors. 


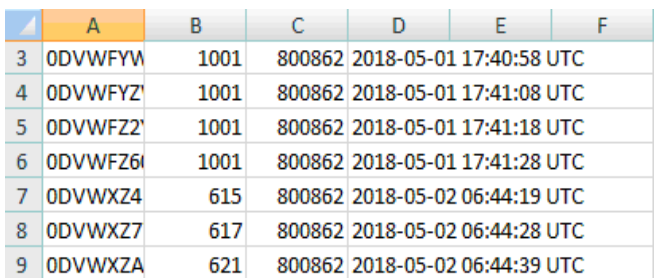

Fig. 20 Sensor 3 Excel sheet Data

\begin{tabular}{|c|c|c|c|c|}
\hline 4 & A & B & C & D \\
\hline 3 & ODVWFYM & 152 & 800863 & 2018-05-01 17:40:58 UTC \\
\hline 4 & ODVWFYZ & 151 & 800863 & 2018-05-01 17:41:08 UTC \\
\hline 5 & ODVWFZ2 & 151 & 800863 & 2018-05-01 17:41:18 UTC \\
\hline 6 & ODVWXZ4 & 348 & 800863 & 2018-05-02 06:44:19 UTC \\
\hline 7 & ODVWXZ7 & 361 & 800863 & 2018-05-02 06:44:28 UTC \\
\hline 8 & ODVWXZA & 370 & 800863 & 2018-05-02 06:44:39 UTC \\
\hline 9 & ODVWXZD & 370 & 800863 & 2018-05-02 06:44:49 UTC \\
\hline
\end{tabular}

Fig. 21 Sensor 4 Excel Sheet Data

\begin{tabular}{|c|l|r|r|l|}
\hline 4 & ODVWXZ7 & 7 & 800868 & $2018-05-02 ~ 06: 44: 28$ UTC \\
\hline 5 & ODVWXZA & 9 & 800868 & $2018-05-02$ 06:44:39 UTC \\
\hline 6 & ODVWXZD & 6 & 800868 & $2018-05-02$ 06:44:49 UTC \\
\hline 7 & ODVXQG2 & 37 & 800868 & $2018-05-03$ 06:32:01 UTC \\
\hline 8 & ODVXQG3 & 58 & 800868 & $2018-05-0306: 32: 03$ UTC \\
\hline 9 & ODVXQG6 & 78 & 800868 & $2018-05-0306: 32: 13$ UTC \\
\hline 10 & ODVXQG9 & 97 & 800868 & $2018-05-0306: 32: 24$ UTC \\
\hline
\end{tabular}

Fig. 22 Sensor 5 Excel Sheet Data

These data displayed in the excel sheet are also time stamped for further analysis. The critical values of any sensor are to be notified to the concerned person to prevent further damage to the system. To do so each sensor values are bounded by two threshold values both upper and lower that would help in identifying the critical values of the sensors.

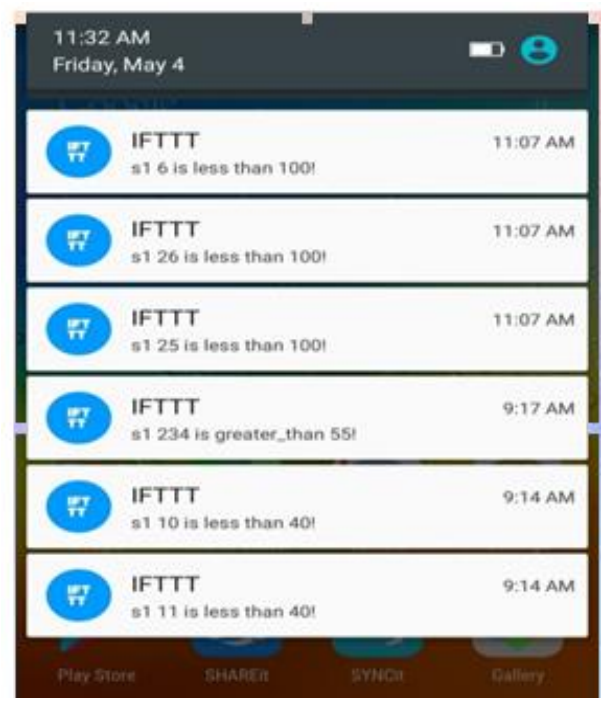

Fig. 23 Notification from IFTTT Server
The figure 23 shows the IFTTT Applet Push message notification in mobile App from all sensors connected to Adafruit io cloud storage with different threshold values set for each sensors.

\section{IMPROVING NODE LIFETIME}

The nodes installed in the wind turbines are battery powered. As each node is connected with number of sensors and WiFi module, it strives hard to withstand the power. The various activities carried out by the nodes such as sensing, processing and communication drains out the battery power. Among these activities transmission of data consumes much power. Data compression is one of the best way in reducing the number of data transmitted and thereby the power consumed during transmission [9]. The life time of the node can be improved by applying a suitable lossless compression algorithm. One such algorithm proposed and used is modified Rice Golomb coding. This algorithm is based on Rice Golomb coding, a lossless compression method that well suits for sensor data compression $[10,11]$.

\subsection{Compression Procedure}

(i) The auto-differencing of the acquired data is taken and find the average of the sequence.

(ii) Find the tuning parameter ' $\mathrm{k}$ ' that is considered to be powers of 2 (i.e. $\mathrm{m}=2^{\mathrm{k}}$ ).

(iii) Divide the average value by ' $\mathrm{m}$ ' for different values of ' $\mathrm{k}$ ' and encode in such a way that remainder is coded in binary and quotient is coded in unary form.

(iv) Optimized ' $\mathrm{k}$ ' is thereby determined by identifying the minimum number of bits used for encoding the average value.

(v) Now this ' $\mathrm{k}$ ' value is used to encode data sequence.

(vi) Encoding format is first eight bits the first data in the original data sequence before auto differencing, next three bits to represent the ' $k$ ' value and is followed by the encoding bits of other data sequence.

\subsection{Decompression Procedure}

(i) From the first 8 bits of the encoded data the first data is identified and from the next three bits ' $\mathrm{k}$ ' value is found.

(ii) Now the reverse process is carried out to decode the data using the ' $\mathrm{k}$ ' value.

(iii) Then with the first data the original data sequence is obtained.

The different parameters acquired from the sensors 
are compressed using the proposed algorithm to reduce the transmission bandwidth and storage space for data in the cloud. Also the energy consumed during transmission is reduced that extends the lifetime of the sensor nodes. As an example the 200 humidity dataset acquired from the humidity sensor is considered for compression. The actual number of bits required to transmit 200 dataset is 1600 bits (i.e. $200 \times 8$ bits). The compression ratio (CR) and space saving Percentage (SP) [18] is given by the formula given below:

$\mathrm{CR}=$ uncompressed size / compressed size

$\mathrm{SP}=[1-($ compressed size/uncompressed size $)] \times 100-(2)$

Therefore after compression the encoded data length is reduced to be 565 bits, compression ratio is 2.83 and the saving percentage is about $65 \%$.

\subsection{Power and Energy consumption Calculation}

With reference to the datasheet of ESP8266 WiFi module [19] it is found that for data rate of $54 \mathrm{Mbps}$ the transmission output power (Pout) is $15 \mathrm{dBm}=$ $0.032 \mathrm{~W}$.

For 1600 bits of humidity dataset before compression the Pout $=0.948 \times 10^{-6} \mathrm{~W}$ and after compression for 565 bits Pout $=0.335 \times 10^{-6} \mathrm{~W}$. This reduction in transmission power consumption after compression indicates the efficient use of energy. Now the energy consumption with respect to power is calculated using the formula given below:

$$
\mathrm{E}_{(\mathrm{kWh} / \mathrm{day})}=\mathrm{P}_{(\mathrm{W})} \times \mathrm{t}_{(\mathrm{h} / \text { day })} / 1000 \mathrm{~W} / \mathrm{kW} \text {----- (3) }
$$

The energy consumption is calculated using the energy consumption calculator [18] before compression with Pout $=0.948 \times 10^{-6} \mathrm{~W}$ to be $2.2752 \mathrm{e}-8 \mathrm{kWh} /$ day and after compression with Pout $=0.335 \times 10^{-6} \mathrm{~W}$ as $8.04 \mathrm{e}-9 \mathrm{kWh} /$ day. Therefore the amount of power in turn energy consumed during the transmission of data is reduced after compression and the power saving obtained for transferring the data from a single sensor is $0.613 \times 10^{-6} \mathrm{~W}$. The result concludes that the lifetime of the node is increased by applying the proposed compression algorithm on the transmitting data.

\section{CONCLUSION}

The proposed system deals with the continuous monitoring of wind turbines and the analysis of data from wind turbine sensors located in the nacelle box. The wind turbine sensor data are transferred to Adafruit IO cloud storage via WiFi module. The push notification is received in mobile phone from IFTTT server when the sensor values deviates from the threshold range. It can be used for data analysis and better communication between wind turbine and control center. The device thereby helps to monitor the physical condition of the wind turbine from anywhere and at anytime. Implementing the proposed compression algorithm on the data from single sensor resulted with the power saving of about $0.613 \times 10^{-6}$ $\mathrm{W}$. Thus by applying the proposed algorithm on the data from all the sensors would result with much more reduction in power consumption. Thereby implementation of the proposed compression algorithm in the system helps to increase the lifetime of the node used for ubiquitous monitoring of wind turbines. In nearby future the wind turbine data communication can be achieved at faster rate with the help of $5 \mathrm{G}$ technology.

\section{REFERENCES}

1. E. Hau, Wind Turbines, Fundamentals, Technologies, Application, Economics, 2nd ed.; Springer: Berlin, Germany, 2006.

2. Henrik Niemann et al., "Fault diagnosis and condition monitoring of wind turbines", International Journal of Adaptive Control and Signal Processing, 24 May 2017.

3. P. Ashwini and R. UmaMaheswari, "Wireless sensor network for condition monitoring of remote wind mill", International Conference on Innovations in Green Energy and Healthcare Technologies (IGEHT), IEEE Xplore: 2nd November 2017.

4. A.R. Al-Ali, "Internet of Things Role in the Renewable Energy Resources", Elsevier, Science Direct, Energy Procedia 100 (2016), 34 - 38.

5. Lian, K. Y., Hsiao, S. J., \& Sung, W. T., "Mobile monitoring and embedded control system for factory environment", Sensors (Switzerland), (2013), 13, 17379-17413.

6. Zhang, Z., Verma, A., \& Kusiak, A., "Fault analysis and condition monitoring of the wind turbine gearbox", IEEE Transactions on Energy Conversion, (2012) 27, 526-535.

7. Nikola Zlatanov, "Arduino and Open Source Computer 
Hardware and Software", IEEE Computer Society, Grant No: VFI Proposal, DOI:10.13140/RG.2.1.1071.7849, 2016.

8. Rishi Kant and Shantanu Bhattacharya, "Sensors for Air Monitoring", Environmental, Chemical and Medical Sensors, Energy, Environment, and Sustainability, Springer 2018.

9. Tetsuya Takezawa, Koichi Asakura and Toyohide Watanabe," Lossless compression of time series data based on increasing average of neighboring signals", electronics and Communication in Japan, Wiley Online library, Volume93, Issue8, August 2010, Pages 47-56.

10. S. Kalaivani and C. Tharini, "Analysis And Modification of Rice Golomb Coding Lossless Compression Algorithm For Wireless Sensor Networks", Journal of Theoretical And Applied Information Technology, 2018.

11. S. Kalaivani and C. Tharini, "Analysis and Implementation of a Lossless Compression Algorithm for Wireless Sensor Networks" Computer Communications, Science Direct, Volume 150, 15 January 2020, Pages 463-471.

12. https://components101.com

13. www.circuitbasics.com/set-bmp180-barometricpressure-sensor-arduino/

14. http://nodemcu.com/index_en.html

15. https://www.arduino.cc/en/Guide/Environment

16. https://learn.adafruit.com/webide/overview

15. https://ifttt.com/

17. https://rechneronline.de/transfer/compression.php

18. https://cdn-shop.adafruit.com/product-files/.../0AESP8266_Datasheet_EN_v4.3.pd.

19. https://www.rapidtables.com/calc/electric/energyconsumption-calculator.html 